Rapid Reviews COVID-19

\title{
Review 1: "Herd immunity thresholds for SARS-CoV-2 estimated from unfolding epidemics"
}

\section{Keith Klugman ${ }^{1}$}

${ }^{1}$ Pneumonia Program Strategy Team, Bill and Melinda Gates Foundation

Published on: Nov 19, 2020

DOI: $10.1162 / 2$ e3983f5.c13af903

License: Creative Commons Attribution 4.0 International License (CC-BY 4.0). 


\section{$\underline{\text { RR:C19 Evidence Scale rating by reviewer: }}$}

- Reliable. The main study claims are generally justified by its methods and data. The results and conclusions are likely to be similar to the hypothetical ideal study. There are some minor caveats or limitations, but they would/do not change the major claims of the study. The study provides sufficient strength of evidence on its own that its main claims should be considered actionable, with some room for future revision.

$* * * * * * * * * * * * * * * * * * * * * * * * * * * * * * * * * * * * * * *$

\section{Review:}

The ascent of SARS-COV-2 has been relentless globally, sparing only a few remote islands that have successfully cut off contact with the rest of the infected planet. While social distancing was shown originally in China to be able to reduce Reff below 1 , this type of social engineering of large populations with unsealed borders has not been possible to the same extent in most other countries. Nonetheless, the four countries analyzed in this model all illustrate the extent to which social distancing can at least temporarily reduce Reff below 1.

Longer term suppression of viral transmission could be aided by herd protection, but as the authors' point out the baseline Ro would only be sufficiently reduced, assuming random mixing, at $60 \%$ or greater levels of both infection and subsequent immunity.

This paper introduces some profound and important concepts challenging the conventional model of defining a threshold of herd protection, by assuming that a small fraction of individuals in the community drive most of the infections, but also that they, by virtue of their personal ability to transmit the virus to multiple contacts, are also more likely to become infected, and thus immune.

Their model then suggests that the greater the coefficient of variation, the lower the threshold required to induce herd, given the level of social distancing, even if social distancing itself reduces that coefficient of variation (extended Fig 5).

These data are demonstrated to fit the existing patterns of the epidemic in those countries, but can only be confirmed as social distancing is relaxed and numbers fail to rise again. So far, the data support this observation but the authors now have access to many far more persuasive settings which they could discuss and which largely, in the view of this reviewer, support their hypothesis. 
Sweden has had reduced mobility despite relaxed political decrees and saw continued transmission far beyond the other Nordic countries yet the epidemic there has peaked, suggesting that herd protection may have played a role.

African countries have been far less able to suppress transmission for obvious economic reasons, yet even in South Africa the rise in infections has plateaued at around the level of exposure suggested by this model.

Some additional data on serological evidence to support the levels of exposure in these countries could be added to the discussion.

This reviewer is not able to comment on the veracity of the statistical analysis in the paper, but the concepts modeled and reported in this paper offer the most compelling model I have seen to date of the epidemic, and suggest that herd protection may be in play and support reduced viral transmission in addition to social distancing in most countries even before effective vaccines become available. 